\title{
Femtosecond Charge Transfer Dynamics of a Modified DNA Base: 2-Aminopurine in Complexes with Nucleotides
}

\author{
Torsten Fiebig, Chaozhi Wan, and Ahmed H. Zewail*a]
}

As a fluorescent isomer of adenine, 2-aminopurine (Ap) is a powerful probe of DNA dynamics and DNA-mediated charge transfer processes. Here, we report studies with femtosecond resolution of the excited-state dynamics of $A p$ in various solvents and in bimolecular complexes with nucleotides. Using timeresolved transient absorption and fluorescence up-conversion methods we identify charge transfer as the origin for the quenching of the Ap fluorescence by all four DNA nucleotides. The direction of the redox process is, however, dependent on the base, and from the rates we deduce the nature of the transfer, hole versus electron

Because of its structural similarity to adenine $(A)$, together with its fluorescence properties and base pairing with thymine ${ }^{[1-3]}$ and even cytosine, ${ }^{[4-6]}$ 2-aminopurine (Ap; Scheme 1) has been incorporated as a sensor base into various DNA assemblies. It<smiles>Nc1ncnc2c1NCN2</smiles>

A<smiles>Nc1ncc2nc[nH]c2n1</smiles>

Ap

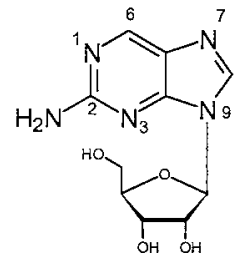

Ap-R
Scheme 1. Structures of adenine (A), 2-aminopurine (Ap), and ribose-substituted 2-aminopurine $(A p-R)$.

has been shown that the replacement of A by Ap may not seriously disrupt the biological interactions of DNA: Ap-containing oligonucleotides (decamers) are still recognized and cleaved by endonucleases and form B-type helix structures, as suggested by NMR studies. ${ }^{[2,4,5]}$ Further reports on such biologically active Ap-containing oligonucleotides have appeared recently. ${ }^{[7,8]}$

The lowest excited singlet state of $\operatorname{Ap}\left(A p^{*}\right)$, which has a $\pi-\pi^{*}$ character ${ }^{[9]}$ is shifted to longer wavelengths compared to those of the natural nucleobases allowing selective excitation of Ap in DNA. Time-resolved fluorescence measurements of Ap in oligonucleotides have been carried out mainly as a tool to probe local and global changes of DNA conformations. ${ }^{[2,10-12]}$ It has been reported that the fluorescence lifetime of $\mathrm{Ap}^{*}$ depends strongly on the microscopic environment. Hence the fluores- transfer. The $\mathrm{pH}$ and the kinetic isotope effects of these charge transfer reactions revealed no evidence for proton transfer involvement in the rate-determining step. From the measured rates and using electron transfer theory we estimate the driving force for charge transfer between all four nucleobases and Ap. The results are important for the studies of dynamics using $A p$ in DNA assemblies.

\section{KEYWORDS:}

2-aminopurine - charge transfer - DNA - femtochemistry

nucleotides

cence decay was found to be multiexponential due to structural and dynamic heterogeneity in DNA. ${ }^{[11,13,14]}$

Recently, Ap has been employed as an electron/hole donor in various DNA assemblies. ${ }^{[14-16]}$ In our earlier report ${ }^{[14]}$ we briefly discussed control experiments involving $A p$ which are relevant to our studies of the femtosecond ( $\mathrm{fs}$ ) dynamics in DNA systems. In this paper, we give a full account of our experimental studies of the ultrafast excited-state dynamics of Ap in different environments, including experiments with $A p$ and $A p$ in complexes with mononucleotides of guanine $(G, d G T P)$, adenine (A, dATP), cytosine (C, dCTP), thymine (T, dTTP), 7-deazaguanine $(Z, d Z T P)$, and inosine (l, dITP) in aqueous/buffer solutions. To elucidate the dynamics of these complexes on the ultrafast time scale we carried out fs-transient absorption and fs-fluorescence up-conversion measurements of $\mathrm{Ap}$ a) in different solvents, b) at different $\mathrm{pH}$ values, and $\mathrm{c}$ ) with nucleotides at different concentrations. We also studied the isotope effect on the dynamics. Finally, we considered the possibility of a ground-state equilibrium between the $7 \mathrm{H}$ and $9 \mathrm{H}$ tautomers of $\mathrm{Ap}$ and carried out a number of control experiments on $A p-R$, a derivative of $A p$ where the proton in the 9-position is replaced by a ribose unit (Scheme 1).

[a] Prof. A. H. Zewail, Dr. T. Fiebig ${ }^{[+]}$, Dr. C. Wan Laboratory for Molecular Sciences

Arthur Amos Noyes Laboratory of Chemical Physics

California Institute of Technology

Pasadena, CA 91125 (USA)

${ }^{+}{ }^{+}$Present address:

Institute for Physical and Theoretical Chemistry

Technical University of Munich

Lichtenbergstrasse 4, 85748 Garching (Germany) 
From these studies, we were able to clearly resolve a biphasic behavior of the $\mathrm{Ap}^{*}$ decay, with a fast component present in all six Ap-nucleotide complexes. Excited-state charge transfer is the dominant mechanism for the depopulation of Ap-nucleotide complexes. Moreover, we characterized the nature of the charge carrier (electron versus hole) by relating the observed rates to estimated values of $\Delta G$. In a recent work by Rachofsky et al., ${ }^{[13]}$ two different mechanisms were proposed for the deactivitation of $\mathrm{Ap}^{*}$, so-called "static" and "dynamic" quenching. However, this distinction depends on the actual time scale of the experiment and, as discussed below, the two cases, groundstate complexes versus excited-state quenching, can be easily distinguished. Such complexes play a crucial role in the studies of DNA assemblies. ${ }^{[17,18]}$

\section{Experimental}

Femtosecond Laser Apparatus: Figure 1 gives a schematic of the experimental setup used in the fs-transient absorption and fluorescence up-conversion measurements. Femtosecond pulses (80 fs, $\sim 800 \mathrm{~nm}, 2 \mathrm{~mJ}$ at $1 \mathrm{kHz}$ ) were generated using a Ti:sapphire laser. The $2 \mathrm{~mJ}$ pulse was split equally to pump two optical parametric amplifiers (OPAs). The signal output from one OPA was then doubled or mixed with the residual $800 \mathrm{~nm}$ pulse to generate the probe pulse at $480-700 \mathrm{~nm}$. The pump pulse at $325 \mathrm{~nm}$ was generated by quadrupling the signal pulse from another OPA (first doubling the signal to $650 \mathrm{~nm}$ and then doubling the latter to $325 \mathrm{~nm}$ ). The pump pulse was passed through an optical arrangement that was controlled by a computer for time delay scans. A chopper was used to optimize the signal-to-noise ratio of the pump pulse. $A \lambda / 2$ wave plate (combined with a polarizer) reduced the pump pulse energy to $0.2 \mu \mathrm{J}$. The probe pulse was attenuated to $<0.01 \mu \mathrm{J}$ by neutral density filters. The polarization of the probe pulse was set at $54.7^{\circ}$ (magic angle), relative to the polarization of the pump pulse.
The fluorescence up-conversion setup is shown in the inset of Figure 1. The fluorescence was initiated by the pump pulse in the sample cell, then collected and focused into a BBO crystal (thickness $1 \mathrm{~mm})$ by two parabolic mirrors. A probe pulse $(\sim 800 \mathrm{~nm}, 100 \mu \mathrm{J})$ was then sent into the $\mathrm{BBO}$ crystal for mixing with the fluorescence signal. The up-converted signal was detected by a photomultiplier after passage through a double-grating monochromator. Before the monochromator, a filter ( $F$, consisting of a grating and mirrors) was used to reduce the background. The pump polarization was fixed at $54.7^{\circ}$ (magic angle) relative to the fluorescence polarization set by the BBO crystal. The signal from the photomultiplier was finally processed by means of a boxcar integrator and a computer. The measurements were carried out in a $5 \mathrm{~mm}$ quartz cell which was stirred during the data collection process.

Treatment of the Data: The fs transients were analyzed using a leastsquares fitting program. All decays in both transient absorption and fluorescence up-conversion were well described by the sum of two exponential functions. To account for the finite experimental response, the exponential decay terms were convoluted with a Gaussian response function $g(\tau)=1 /\left(\pi^{1 / 2} \sigma\right) \cdot \exp \left[-(t / \sigma)^{2}\right]$. The resulting time-dependent signal $S(t)$ is given by Equation (1), which can be solved analytically to give Equation (2), where erf is the error function and $c_{i}$ is the amplitude of the component $i$ with decay time $\tau_{i}$. The term $\sigma$ is related to the full width at half maximum (FWHM) of the experimental response $(\sigma=\mathrm{FWHM} / 1.6651)$.

$$
\begin{aligned}
& S(t)=c_{1} \int_{-\infty}^{t} g(\tau) \exp \left(-\frac{t-\tau}{\tau_{1}}\right) \mathrm{d} \tau+c_{2} \int_{-\infty}^{t} g(\tau) \exp \left(-\frac{t-\tau}{\tau_{2}}\right) \mathrm{d} \tau \\
& S(t)=\sum_{i=1}^{2} c_{i} \cdot \frac{1}{2} \cdot \exp \left[\left(\frac{\sigma}{2 \tau}\right)^{2}-\frac{t}{\tau_{\mathrm{i}}}\right] \cdot\left[1-\operatorname{erf}\left(\frac{\sigma}{2 \tau_{\mathrm{i}}}-\frac{t}{\sigma}\right)\right]
\end{aligned}
$$

The quality of the fits were judged using the $\chi^{2}$ criteria. We estimated the error to be $\sim 10 \%$ of the short decay time components. The decay components in the up-conversion experiment have an error range of $\sim 20 \%$.

Materials: Ap, 2-aminopurine-9-ribofuranoside (Ap-R), thymidine, 3-methylthymidine, and all monophosphate nucleotides (all Sigma-Aldrich) and the triphosphate nucleotides (Pharmacia Biotech) were purchased. Experiments were carried out at room temperature in water $(\mathrm{pH} 7)$ if not stated otherwise. In all experiments we used an Ap concentration of $\sim 10^{-4} \mathrm{M}$.

\section{Results}

Figure 2 shows the transient absorption decays of Ap after excitation into its $S_{1}$ state, in the presence of six different nucleotides in aqueous solution. All transients exhibit clearly biexponential decay. The fast decay components $\left(\tau_{1}\right)$ are strongly dependent on the nature of the nucleotide and show a dramatic difference of two orders of magnitude ranging from 2.7 (Ap - dZTP) to $350 \mathrm{ps}$ (Ap - dATP). In contrast, the slow decay components $\left(\tau_{2}\right)$ are longer than $1 \mathrm{~ns}$ for all nucleotide systems except for Ap-dZTP $\left(\tau_{2}=\right.$ $130 \mathrm{ps})$. Note that in the absence of the nucleotides, $\mathrm{Ap}^{*}$ exhibits a decay on the time scale of $\sim 10 \mathrm{~ns}$ (see Figure 4 below). The amplitudes of the picosecond (ps) components depend strongly on the 

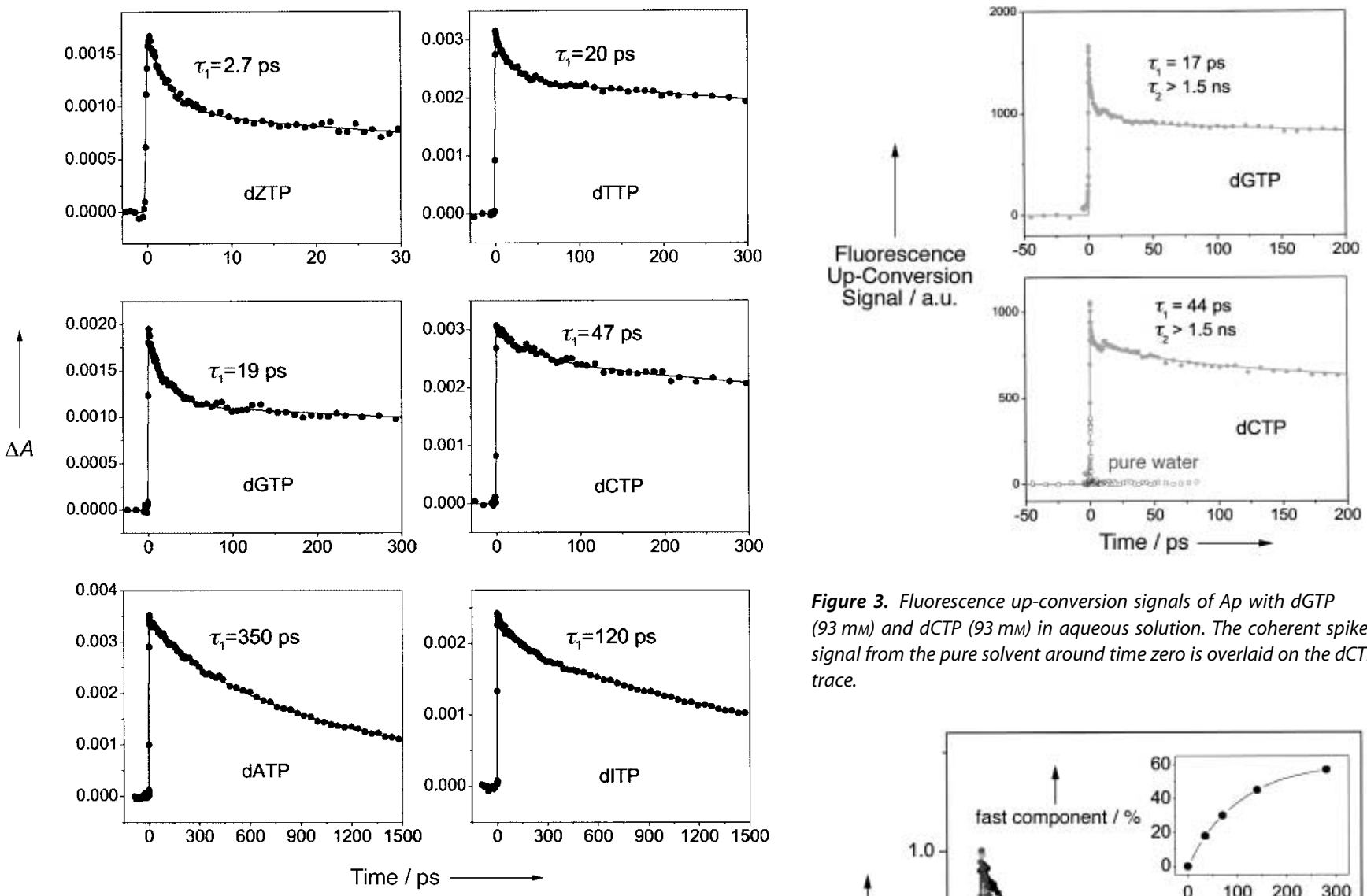

Figure 3. Fluorescence up-conversion signals of Ap with dGTP (93 $\mathrm{mm}$ ) and dCTP (93 $\mathrm{mm}$ ) in aqueous solution. The coherent spike signal from the pure solvent around time zero is overlaid on the dCTP trace.

Figure 2. Time-resolved transient absorption of $A p$ in aqueous solutions with dZTP $(70 \mathrm{~mm})$, dGTP (86 mM), dATP (75 mM), dTTP (75 mM), dCTP (75 mm), and dITP (75 mM). The fast component of a biexponential fit function is characterized by a decay time $\tau_{1}$. Note the change in time scales.

nature of the nucleotide and vary between 16\% (dITP) and $45 \%$ (dZTP). Replacing Ap by Ap-R leads to similar results in the dynamics (data not shown). ${ }^{[19]}$

To ensure that the decay of the transient absorption signal is that of the initially excited Ap* population, we also performed fsfluorescence up-conversion measurements. In Figure 3 we show the up-conversion data of Ap/dGTP and Ap/dCTP systems. The up-conversion measurements give the same fast decay components ( $\tau_{1}=17$ and $44 \mathrm{ps}$ for Ap/dGTP and Ap/dCTP) similar to that observed in the transient absorption $\left(\tau_{1}=19\right.$ and $47 \mathrm{ps}$ ) within the experimental error. The slow decay component has a lifetime of $>1.5 \mathrm{~ns}$. However, the up-conversion signals have a larger noise level and lower accuracy compared to the transient absorption data. In addition, a coherent spike around time zero, which is caused by the solvent (see Figure 3), makes the evaluation of the fluorescence data less precise. In the following we will therefore discuss the transient absorption data only. It should also be noted that monophosphate nucleotides and triphosphate nucleotides, as well as nucleosides, exhibit basically the same behavior within our experimental accuracy.

To investigate the origin of the two decay components we studied the dependence of the fast components on the concentration of the nucleotides. Figure 4 shows the transients of Ap with different dGTP concentrations ranging from 0 to

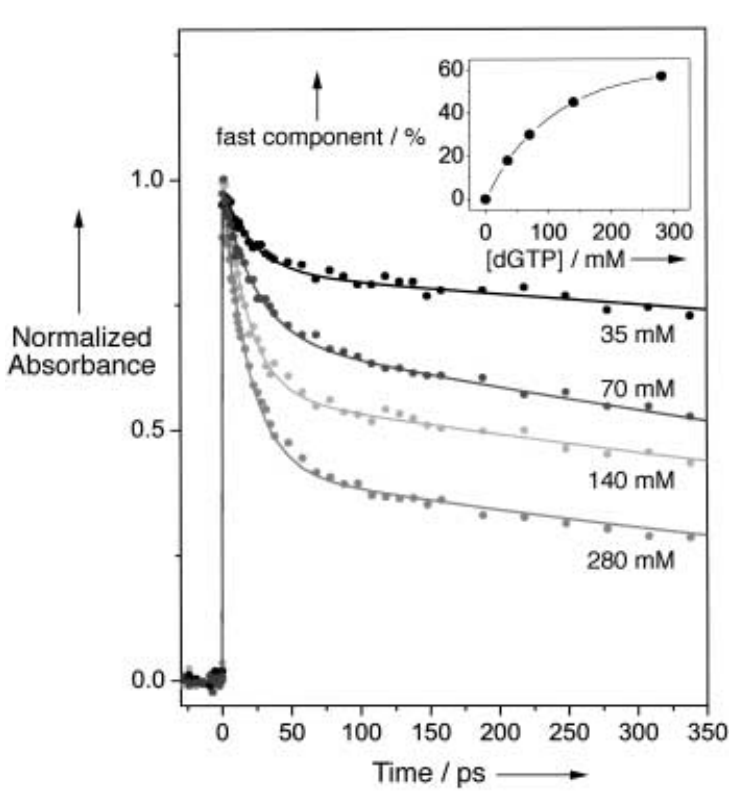

Figure 4. Normalized transient absorption of Ap/dGTP for different concentrations of dGTP. Inset: The fast component percentage versus the concentration of dGTP; the line is a fit based on a kinetic model for a 1:1 complex formation and provides the equilibrium constant for the formation of the complexes (static quenching); dynamic quenching is on a much longer time scale than that of charge transfer, see text.

$280 \mathrm{~mm}$. Although the ps decay rate remains constant, its amplitude increases upon increasing concentration of dGTP. This behavior is well known and indicates the presence of a groundstate complex of Ap and the nucleotide. Thus, the fast decay component reflects the excited-state lifetime of the complex Ap/G while the slow component is attributed to the free (uncomplexed) Ap* population, including any dynamic quenching of Ap*. Evidence for complex formation is given by the good correlation between the amplitude of the fast component and 
the dGTP concentration when analyzing the data in the framework of a kinetic model for 1:1 complex formation $(A+B \Leftrightarrow A-B$; see Figure 4 inset). The corresponding association constant was determined to be $K \approx 8.3 \mathrm{M}^{-1}$; the concentration of the complex increases while the concentration of free Ap decreases with increasing concentration of dGTP. The nanosecond (ns) decay component $\left(\tau_{2}\right)$ reflects, therefore, the decay of the free Ap* and any dynamic quenching; a diffusion-controlled process $\left(10^{9}-\right.$ $10^{10} \mathrm{M}^{-1} \mathrm{~s}^{-1}$ ) which occurs faster with increasing concentration of nucleotides ("dynamic quenching") is expected to occur on the ns time scale (for a nucleotide concentration of $\sim 0.1 \mathrm{M}$ ). However, these slower dynamics cannot be monitored accurately with our experimental setup. The lifetime of the complex which is independent of the dGTP concentration is represented by $\tau_{1}$. We have obtained similar results for the concentration dependence in all Ap/nucleotide systems studied.

Previous measurements of the steady-state fluorescence of Ap in various solvents ${ }^{[20]}$ have shown that the quantum yield depends on the solvent used; in general it has been found that the fluorescence quantum yield becomes smaller in nonpolar aprotic solvents relative to that in polar protic solvents. Figure 5 shows our measured transients in water, ethanol, dimethyl

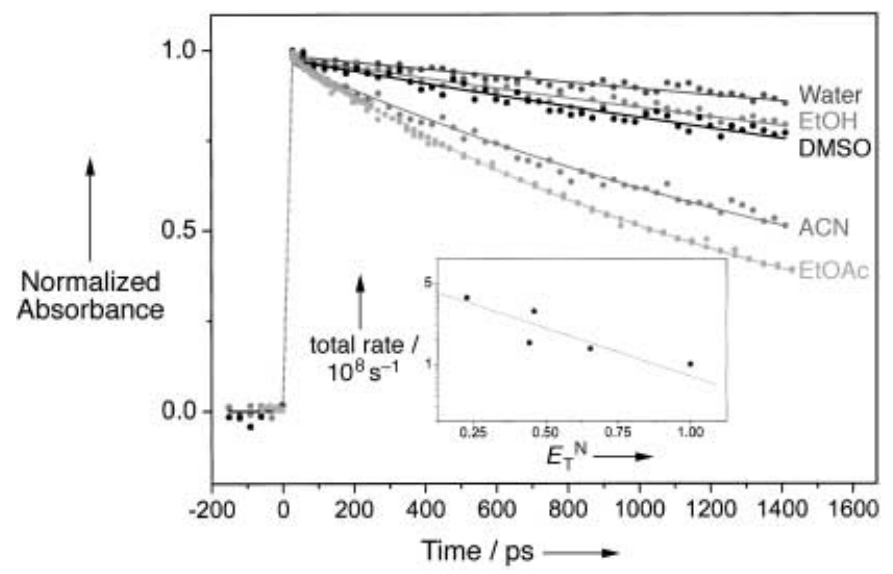

Figure 5. Normalized transient absorption of Ap in different solvents. The transients have been fitted using a single exponential function. Inset: The total rate versus the empirical solvent polarity parameter $\mathrm{E}_{T}^{N}{ }^{[21]}$

sulfoxide (DMSO), acetonitrile, and ethyl acetate, where the empirical solvent polarity parameter $E_{T}^{N}$ changes from 1.0 to 0.228 . ${ }^{[21]}$ The lifetime of $A p^{*}$ varies strongly with the solvent, but a ps component-as observed in the nucleotide complexeswas not found. All these transients show decays with lifetimes ranging from $\approx 1.5 \mathrm{~ns}$ in ethyl acetate to $\sim 10 \mathrm{~ns}$ in water. The results indicate that the lifetime is longer in polar protic solvents and becomes shorter in nonpolar aprotic solvents. In fact, the total decay rate of $\mathrm{Ap}^{*}$ correlates with $E_{\mathrm{T}}^{\mathrm{N}[21]}$ as shown in the inset of Figure 5.

It has been reported previously that Ap can undergo proton transfer, namely deprotonation in basic solvents in the excited state but not in the ground state. ${ }^{[20]}$ We carried out transient absorption measurements to observe the deprotonation dynamics of $\mathrm{Ap}^{*}$ in two basic media: aqueous $\mathrm{NaOH}$ and pure pyridine. The decay rate of the process depends strongly on the basicity of the medium and on the concentration of the base molecules (the basicity of $\mathrm{OH}^{-}$is much higher than that of pyridine $\left.\left(\mathrm{p} K_{\mathrm{a}}=5.6\right)\right)$. The measured transients show the decay for Ap in $1 \mathrm{M} \mathrm{NaOH}$ solution and pure pyridine with lifetimes of 110 ps (data not shown) and 250 ps (Figure 6), respectively.

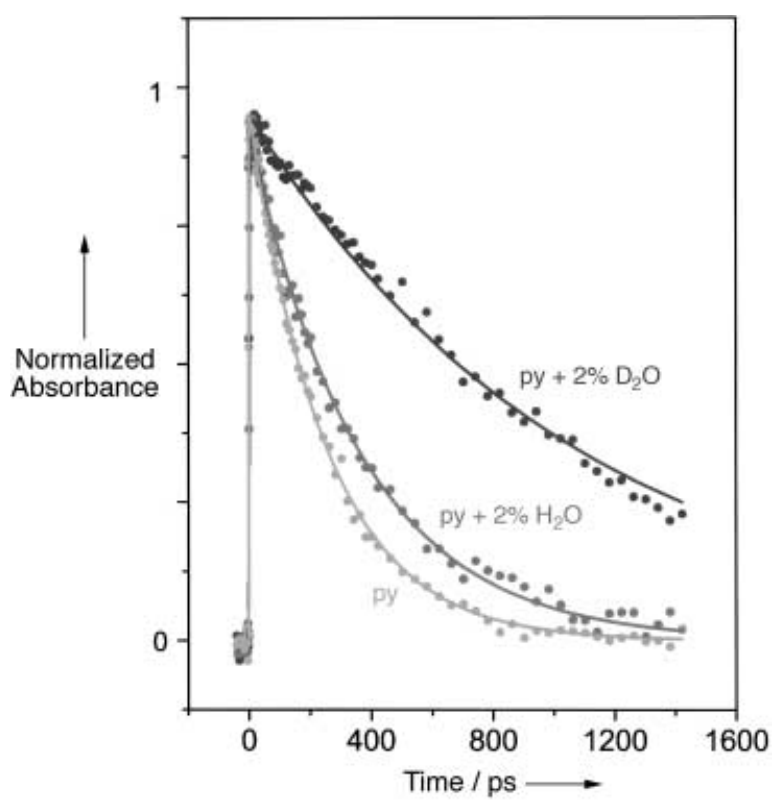

Figure 6. Normalized transient absorption of Ap in pure pyridine (py), pyridine with $2 \% \mathrm{H}_{2} \mathrm{O}$, and pyridine with $2 \% \mathrm{D}_{2} \mathrm{O}$. The solid lines are the fits to a single exponential function.

In order to study the isotope effect on the deprotonation of $\mathrm{Ap}^{*}$ we carried out the following experiments in binary solvent mixtures. We measured the decay of $\mathrm{Ap}^{*}$ in a pyridine solution with $2 \% \mathrm{H}_{2} \mathrm{O}$ added (see Figure 6). As expected, the lifetime increases (from $250 \mathrm{ps}$ in pure pyridine to $370 \mathrm{ps}$ in mixtures with $2 \% \mathrm{H}_{2} \mathrm{O}$ ) as a result of the change in the polarity. If we, however, used a solvent mixture of $2 \% \mathrm{D}_{2} \mathrm{O}$ in pyridine, the lifetime of $\mathrm{Ap}^{*}$ increased to $\sim 1 \mathrm{~ns}$. In the latter case, some of the Ap protons are in situ replaced by deuterons. It is known that the protons of the amino group exchange quickly (on the time scale of $1 \mathrm{~ms}$ to $1 \mathrm{~s}$ ) in $\mathrm{D}_{2} \mathrm{O} .^{[22]}$ This striking result demonstrates that the lifetime of $\mathrm{Ap}^{*}$ is sensitively influenced by isotopic substitution but such effects involving possibly the deprotonation occur on longer time scales than those involved in the charge transfer reactions.

For the complexes with the nucleotides this conclusion was confirmed by carrying out two control experiments: a) We varied the $\mathrm{pH}$ value in water from 5.5 to 10.5 , and b) we measured the isotope effect on the dynamics by changing the solvent from $\mathrm{H}_{2} \mathrm{O}$ to $\mathrm{D}_{2} \mathrm{O}$. Figure 7 shows the $\mathrm{pH}$ dependence of the transient absorption decay in Ap-G and Ap-T. We observed almost no change in the two decay rates and only a small change in their relative amplitudes as the $\mathrm{pH}$ was changed from 5.5 to 10.5 . Consistent with the lack of a significant $\mathrm{pH}$ dependence is the relatively small isotope effect we observed for $A p / G$ and 


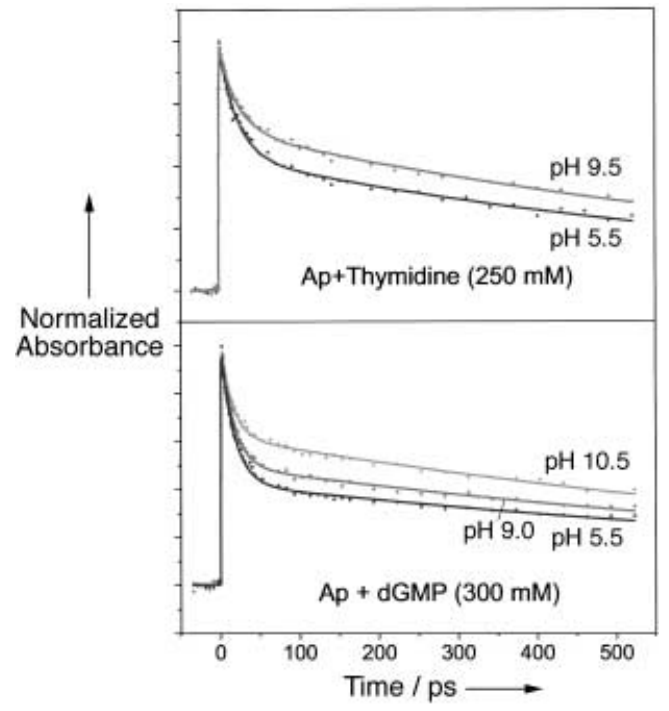

Figure 7. Normalized transient absorption of Ap with thymidine $(250 \mathrm{~mm})$ at pH 5.5 and 9.5 and dGMP (300 mM) at pH 5.5, 9.0, and 10.5 in water.

$\mathrm{Ap} / \mathrm{T}$, shown in Figure 8. However, both systems show quantitative differences in their isotope effects: In Ap/G the amplitude of the fast component decreases from $57 \%$ to $54 \%$ while the decay time $\tau_{1}$ increases from 19 to $30 \mathrm{ps}$. In contrast,

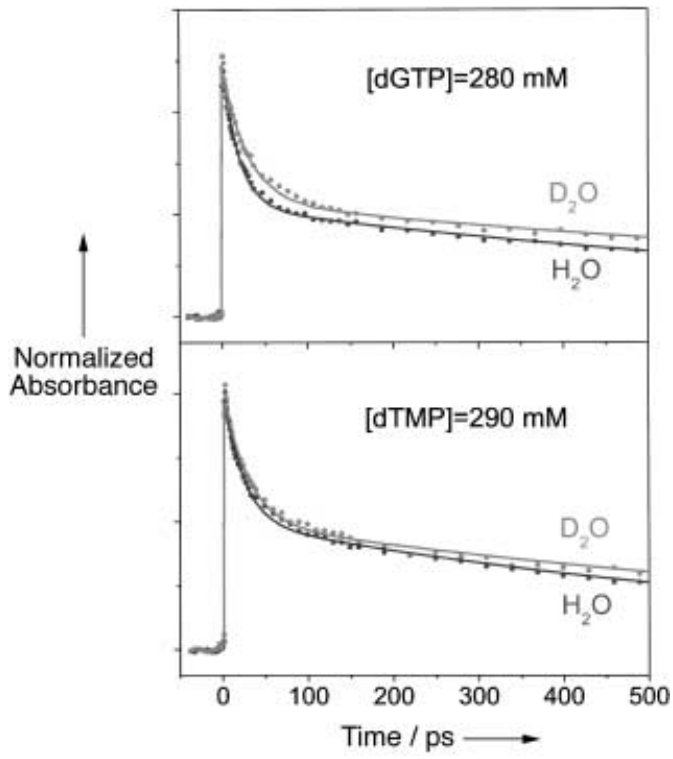

Figure 8. Normalized transient absorption of Ap with dGTP (280 mM) and dTMP (290 mm) in $\mathrm{H}_{2} \mathrm{O}$ and $\mathrm{D}_{2} \mathrm{O}$.

for $\mathrm{Ap} / \mathrm{T}$ no difference in the amplitudes was observed, and only a minor change in the ps decay time from $23\left(\mathrm{H}_{2} \mathrm{O}\right)$ to $29 \mathrm{ps}\left(\mathrm{D}_{2} \mathrm{O}\right)$ was found. Although there have been found differences in the dynamics in both $A p / G$ and $A p / T$, upon changing the solvent from $\mathrm{H}_{2} \mathrm{O}$ to $\mathrm{D}_{2} \mathrm{O}$ a significant involvement of proton transfer cannot be concluded, as discussed in more detail below.

\section{Discussion}

The experimental results reported above reveal four points that are important to emphasize: a) Ap forms complexes with the nucleotides studied, b) the lifetimes of these complexes are short compared to the lifetime of the free $\mathrm{Ap}^{*}$ in water and in various other solvents, c) Ap* can undergo excited-state deprotonation in basic solvents, but d) proton transfer is insignificantly involved on the time scale of our experiment and that the complex structure is robust and it does not change drastically upon varying the $\mathrm{H}^{+}$concentration in the surrounding medium. Based on these experimental observations, in what follows we shall discuss the nature of the ultrafast dynamics and its dependence on the nucleotides. With this in mind we consider the processes of internal conversion, energy transfer, and charge transfer.

\section{Internal Conversion}

$N$-heterocyclic aromatics like Ap often have two low-lying excited states $\left(\pi-\pi^{*}, n-\pi^{*}\right)$ with an energy gap which is sensitive to substitution and solvent effects. Vibronic coupling between these states can open up an efficient nonradiative decay channel into the ground state, the so-called proximity effect or pseudo Jahn - Teller distortion with the consequence of a rapid decay. ${ }^{[23]}$ The magnitude of the coupling is determined by the energy gap between the two low-lying excited states. Small energy gaps can cause strong vibronic coupling, leading to large Franck-Condon factors for nonradiative decays such as internal conversion or intersystem crossing and, hence, to small quantum yields for the competing fluorescence. The energy gap can be sensitively controlled by the surrounding medium. In polar protic solvents, the lower state $\left(\pi-\pi^{*}\right)$ is stabilized by the $\mathrm{H}$-bonding molecules while the upper state $\left(n-\pi^{*}\right)$ is destabilized, leading to a larger energy gap compared to the gas phase or nonpolar solvents. This mechanism has been discussed for Ap to explain the strong dependence of the fluorescence quantum yield on the nature of the solvent. ${ }^{[20]}$ Indeed, quantum chemical calculations on Apwater clusters using a self consistent reaction field approach ${ }^{[24]}$ have shown that solvation and hydrogen bonding can lead to preferential lowering of the $S_{1}$ state and thus the increase in the $\mathrm{S}_{1}-\mathrm{S}_{2}$ gap. While these vibronic coupling effects are important, the total rate of nonradiative decay could be significantly influenced by the presence of conical intersections of potential energy surfaces involving the ground state. ${ }^{[25]}$

Finally, it is interesting to note that we also investigated Ap complexes with a derivative of thymine (3-methylthymidine) where the only $\mathrm{H}$-atom that could act as a $\mathrm{H}$-donor to $\mathrm{Ap}$ (within the hydrogen-bonding framework) is replaced by a methyl group. The fact that we observed the typical ps component in this system indicates that the dynamics are not very sensitive to structural changes. It also suggests that they are not caused by specific hydrogen-bonding interactions between Ap and the bases. Based on the range of the observed dynamics ( $\sim 1$ to $20 \mathrm{~ns}$ ) in various chemically different solvents, it seems unjustified to assume that similar "solvent effects" could account for the ps dynamics observed in the Ap-nucleotide complexes. 


\section{Energy Transfer}

The mechanism of energy transfer can also be excluded because of the lack of overlap between the fluorescence spectrum of $A p^{*}$ and the absorption spectra of the nucleotides. Besides, no change in the fast decay rate was observed upon varying the concentration of the nucleotides. Note that in previous reports on energy transfer in Ap-containing systems $s^{[26,27]}$ the DNA bases have been initially excited (around $270 \mathrm{~nm}$ ) and subsequent transfer of excitation energy to Ap was monitored by steadystate spectroscopy.

It should be mentioned that we also carried out steady-state fluorescence measurements at longer emission wavelengths to detect emission from the excited state of the complex. Such exciplex-type emission could originate from a locally excited state (for example $A p^{*}-G$ ) mixed with partial charge transfer character (which is mainly responsible for the red-shift of the emission). However, even at high concentration of the nucleotide $(\sim 500 \mathrm{~mm})$ no apparent exciplex emission could be detected.

\section{Charge Transfer}

The driving force for charge transfer is determined by the difference of the reversible redox potentials of the donor and acceptor. Although, there is no complete set of reversible potentials of the DNA bases and Ap, some information is already available in the literature. The peak potential for one-electron reduction of $\mathrm{Ap}^{*}$ has been determined to be $+1.5 \mathrm{~V}$ (versus $\mathrm{NHE}) .{ }^{[15]}$ According to this value, $A p^{*}$ should be able to oxidize $Z$ $(+1.0 \mathrm{~V}){ }_{r^{[28]}} \mathrm{G}(+1.29 \mathrm{~V}){ }^{[29]}$ and maybe even $\mathrm{A}(+1.42 \mathrm{~V}) .^{[29]}$ The reverse process, electron transfer (ET) from $A p^{*}$ to a DNA base requires knowledge of the oxidation potential of $A p$, which has not yet been obtained as a reversible signal in the cyclic voltammetry. Shafirovich et al. have estimated a value of $+1.34 \mathrm{~V}$ for $E\left(\mathrm{Ap}^{*+} / \mathrm{Ap}\right)$. $^{[30]}$ Given an excited-state energy for $\mathrm{Ap}^{*}$ of $E_{00}=$ $3.74 \mathrm{eV}$, it becomes apparent that $A p^{*}$ can reduce any base with a reduction potential of about $-2.5 \mathrm{~V}$ or higher (namely, a smaller absolute value). However, the error of this potential due to irreversible electrochemical follow-up reactions has been estimated to be $\pm 300 \mathrm{mV} \cdot{ }^{[31]}$ Finally, there have been at least two different values reported for the oxidation potential of I $\left(+1.5^{[15]}\right.$ and $\left.1.39 \mathrm{~V}^{[32]}\right)$ but no values for the reduction potential. Therefore, it is not possible to obtain accurate and conclusive $\Delta G$ values for either the reduction (ET) or the oxidation processes (hole transfer, HT) of the nucleobases. Moreover, in the case of I we can only speculate whether HT or ET operates. ${ }^{[33]}$

Despite the uncertainties related to irreversible electrochemistry, a trend for the oxidizability of the nucleobases has been established: $Z>G>A \gg C>T^{[29,34]}$ This trend is supported by correlation of peak potentials with measured ionization potentials in the gas phase $\mathrm{e}^{[35]}$ and by high-level DFT calculations of the adiabatic ionization potentials. ${ }^{[36]}$ However, our experiments on the fs time scale do not follow this trend. We found almost the same rate constants for $\mathrm{Ap} / \mathrm{G}$ and $\mathrm{Ap} / \mathrm{T}$ but much slower rates for $\mathrm{Ap} / \mathrm{A}$ and $\mathrm{Ap} / \mathrm{I}$. It is important to note that reduction properties of the nucleobases are far more difficult to characterize, in both experiment and theory, than the oxidation properties. Recent studies of electron affinities ${ }^{[36,37]}$ of the bases are in agreement with an earlier trend established for the reducibility of nucleobases based on electrochemical methods: ${ }^{[34]} \mathrm{T}>\mathrm{C}>\mathrm{A}>\mathrm{G}$. Thus if the ps process in the $\mathrm{Ap}$-nucleotide complexes would involve ET from Ap* to the nucleotide, one would expect the fastest rate for the Ap-T system. Based on the observed dynamics, but entirely consistent with the available set of redox potentials, we therefore conclude that both reaction types (HT and ET), namely reduction and oxidation of nucleotides by $\mathrm{Ap}^{*}$, are present in our series of complexes.

The direction of charge transfer for Ap/Z,Ap/G,Ap/T, and $A p / C$ is the following: $Z$ and $G$ are oxidized, whereas $T$ and $C$ are reduced. It is expected that $\mathrm{A}$ and I will be positioned somewhere in between these two limits, maybe even undergo both HT and ET. It is noteworthy to mention that this interpretation is also supported by a recent theoretical study. Jean et al. have carried out ab initio calculations on the stacked complexes of Ap with nucleobases. ${ }^{[38]}$ Although these calculations neglect environmental effects, such as the solvent or neighboring bases, they clearly revealed the existence of excited states with charge transfer character for all four DNA bases. In particular for Ap/T complexes, a distinct energetically low-lying charge transfer state was obtained when an electron was transferred from Ap to T.

From an electrochemical point of view, the absence of a $\mathrm{pH}$ dependence in our transients may appear unusual, since measurements of (reversible) redox potentials exhibit a strong $\mathrm{pH}$ dependence. ${ }^{[34,39]}$ In parallel one would expect a change in the decay rate with the $\mathrm{pH}$ if equilibrium is established. The striking fact that our experiments, in contrast to the electrochemistry, do not show a $\mathrm{pH}$ dependence suggests that charge transfer is the initial step of the reaction sequence and that other follow-up reactions, which may lead to equilibration, occur on a much longer time scale. These slower processes make the charge transfer on the surface of the electrode irreversible and cause shifts in the peak potential upon changing the $\mathrm{pH}$ value, as reported. ${ }^{[40]}$ This picture is consistent with a report on the lifetime of Ap radical cations in aqueous solution $(\sim 30 \mathrm{~ns})^{[41]}$ which undergo deprotonation.

The measured rates of charge transfer can be inverted to obtain $\Delta G$ using the Marcus-type expression. ${ }^{[42]}$ We assumed a typical reorganization energy for aqueous solutions of $\lambda=$ $1.2 \mathrm{eV}^{[34,43]}$ and an electronic coupling matrix element of $V=$ $100 \mathrm{~cm}^{-1}$ for both the reduction and oxidation. The calculated rates were compared with our experimental lifetimes in order to estimate $\Delta G$ for both $\mathrm{HT}$ and ET processes. The results are shown in Figure 9. In this picture, the oxidative (HT) and reductive (ET) regime are shown on the left- and right-hand sides. The dashed line is the theoretical Marcus curve. Based on this analysis, Z, G, and $A$ are oxidized by $A^{*}$ while $T$ and $C$ are placed in the reduction branch. Note that if $T$ and $C$ were also oxidized, the rates would be orders of magnitude slower than the ones observed. For HT we obtained free energies of $-0.73(\mathrm{Z}),-0.53$ $(\mathrm{G})$, and $-0.3 \mathrm{eV}(\mathrm{A})$. The corresponding redox potentials from Barton et al. ${ }^{[15,28]}$ and Steenken et al. ${ }^{[29]}$ give somewhat smaller driving forces, approximately $-0.5(\mathrm{Z}),-0.3(\mathrm{G}),-0.1(\mathrm{~A})$, and 


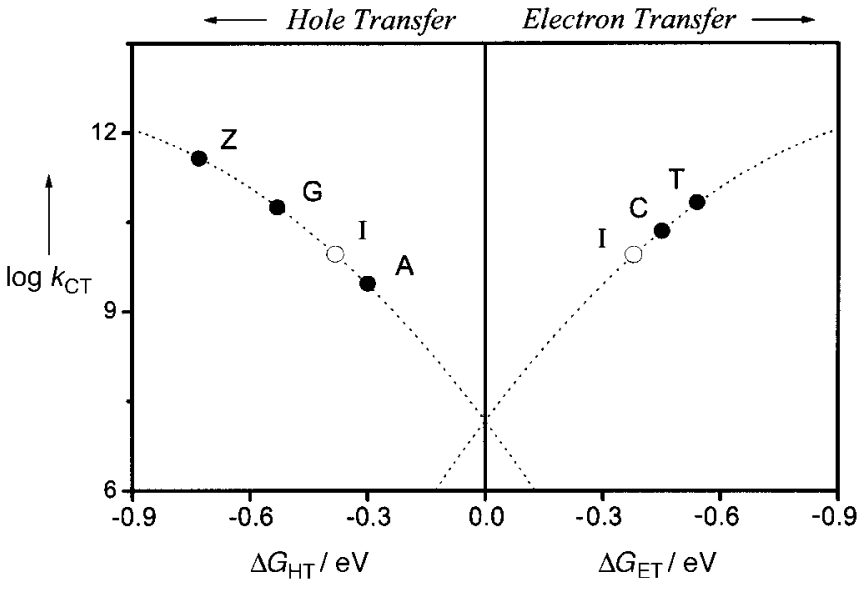

Figure 9. Plot of the estimated driving force $\Delta \mathrm{G}$ for electron and hole transfer, based on measured rates. The dashed line is a theoretical curve obtained by using the Marcus equation (see text), assuming a reorganization energy of $\lambda=1.2 \mathrm{eV}$ and an electronic coupling matrix element $V=100 \mathrm{~cm}^{-1}$. The data points for $Z, G$, and $A$ are shown in the oxidation channel (left side) and the data points for $T$ and $C$ are shown in the reduction channel (right side). Note that for I (open circle) a clear assignment of the redox direction is not possible (see text for details).

$0.0 \mathrm{eV}(\mathrm{I})$; however, the differences are within the experimental error, in particular due to the irreversibility of the electrochemical processes. Besides, our $\Delta G$ values for $E T$ from $A p^{*}$ to $C(-0.45 \mathrm{eV})$ and $\mathrm{T}(-0.54 \mathrm{eV})$ are close to the values one obtains by using the oxidation peak potential for $\mathrm{Ap}$ and the reduction potentials from Seidel et al.. ${ }^{[34]} \sim-0.3 \mathrm{eV}$ for $\mathrm{C}$ and $\sim-0.4 \mathrm{eV}$ for $\mathrm{T}$.

Although the measured isotope effect for charge transfer is relatively small, the dependence on the nature of the nucleotide may be significant. The isotope effect is more pronounced in the complexes with purine bases $(Z, G$, and $A)$ than in those with pyrimidine bases ( $C$ and $T$ ). However, this does not necessarily implicate that proton transfer is coupled to the charge transfer process. One has to be cautious with this interpretation for a number of reasons. First, besides changing the acidic protons in the solute (Ap and nucleobases) the reaction also occurs in a different solvent $\left(\mathrm{D}_{2} \mathrm{O}\right.$ instead of $\left.\mathrm{H}_{2} \mathrm{O}\right)$. The reaction dynamics in the complex are influenced by the nature of the solvent, particularly by its hydrogen-bonding capabilities. $\mathrm{D}_{2} \mathrm{O}$ and $\mathrm{H}_{2} \mathrm{O}$ are known to be different in this respect. ${ }^{[4,45]}$ Therefore, changes in the reorganization energy and/or in the driving force are expected when using $\mathrm{D}_{2} \mathrm{O}$ instead of $\mathrm{H}_{2} \mathrm{O}$. Similar results on charge transfer systems where nucleobases were involved have been reported by Shafirovich et al. ${ }^{[46,47]}$ Based on these considerations one might even expect a larger isotope effect for purine bases since they provide more nitrogen atoms with solventinteracting lone-pair electrons.

We performed DFT calculations ${ }^{[48]}$ on the neutral guanine and its radical cation, which show that the amino group forces planar geometry in the radical cationic state to allow additional charge to flow from the nitrogen lone-pair into the aromatic ring system. This "umbrella motion" around the amino nitrogen, if coupled to the charge transfer process, could also account for the observed isotope effect. DFT calculations on cytosine (and its radical anion) have also revealed structural changes involving the amino group: Here, the amino group is strongly decoupled (pyramidal) from the aromatic ring in the radical anion. This result has also been reported by Wetmore et al. ${ }^{[36]}$ who applied a higher level of theory. In both cases (oxidation of $G$ and reduction of $\mathrm{C}$ ) the amino groups undergo large structural changes upon charge transfer. Generally, an isotope effect will be expected if these structural reorganizations are coupled to the transfer process even in systems where proton transfer can be excluded. ${ }^{[49,50]}$ Consistent with our interpretation is the fact that we observed the smallest isotope effect in the Ap-T system, since thymine does not possess an amino group which could be deprotonated. In order to make more quantitative predictions about the isotope effect, one has to acquire knowledge of the potential energy surface along the umbrella motion in the presence of $\mathrm{H}_{2} \mathrm{O}$ or $\mathrm{D}_{2} \mathrm{O}$ for both moieties (oxidized and reduced form) involved in the complex.

\section{Conclusion}

In summary, we have studied six nucleobase complexes with Ap* elucidating the significance of charge transfer; other nonradiative decay mechanisms, such as energy transfer or proton transfer, are less significant. Our measured rates suggest that both ET and HT are possible mechanisms that have to be considered when Ap is used as a probe base in DNA. From the measured rates we estimated the free energies for both $\mathrm{HT}$ to $\mathrm{G}$ and $A$, and $E T$ to $T$ and $C$. Furthermore, the results imply that some bases ( $A$ and I) may have ambivalent redox properties, and that the actual reaction path could then depend critically on local binding sites and details of the microscopic environment. The characterization of the $\mathrm{Ap}^{*}$ interaction with all DNA bases forms the foundation for studies of charge transfer in DNA using Ap as a chromophore. Since every (natural) nucleobase can be a potential partner for charge transfer with $\mathrm{Ap}^{*}$, one must be cautious with interpreting both the fluorescence quantum yields and the time-resolved data. In an earlier publication ${ }^{[14]}$ we reported studies of $A p$ as a probe of electron transfer rates in DNA assemblies; in a future report will provide a full account of the work, including the effect of the temperature.

This work was supported by the National Science Foundation, and is part of the collaborative effort with Professor Jacqueline Barton's group.

[1] S. M. Watanabe, M. F. Goodman, Proc. Natl. Acad. Sci. USA 1981, 78, 2864.

[2] T. M. Nordlund, S. Andersson, L. Nilsson, R. Rigler, A. Gräslund, L. W. McLaughlin, Biochemistry 1989, 28, 9095.

[3] S. M. Law, R. Eritja, M. F. Gooman, K. J. Breslauer, Biochemistry 1996, 35, 12329.

[4] L. C. Sowers, G. V. Fazakerley, R. Eritja, B. E. Kaplan, M. F. Goodman, Proc. Natl. Acad. Sci. USA 1986, 83, 5434.

[5] L. W. McLaughlin, T. Feong, F. Benseler, N. Piel, Nucleic Acids Res. 1988, 16, 5631.

[6] P.A. Fagan, C. Fabrega, R. Eritja, M. F. Goodman, D. W. Wemmer, Biochemistry 1996, 35, 4026.

[7] B. Holz, S. Klimasauskas, S. Serva, E. Weinhold, Nucleic Acids Res. 1998, 26, 1076.

[8] B. W. Allan, N. O. Reich, J. M. Beechem, Biochemistry 1999, 38, 5308. 
[9] A. Broo, J. Phys. Chem. A 1998, 102, 526.

[10] C. R. Guest, R. A. Hochstrasser, L. C. Sowers, D. P. Millar, Biochemistry 1991, 30, 3271.

[11] R. A. Hochstrasser, T. E. Carver, L. C. Sowers, D. P. Millar, Biochemistry 1994, 33, 11971.

[12] E. L. Rachofsky, E. Seibert, J. T. Stivers, R. Osman, J. B. A. Ross, Biochemistry 2001, 40, 957.

[13] E. L. Rachofsky, R. Osman, J. B. A. Ross, Biochemistry 2001, 40, 946.

[14] C. Wan, T. Fiebig, O. Schiemann, J. K. Barton, A. H. Zewail, Proc. Natl. Acad. Sci. USA 2000, 97, 14052.

[15] S. O. Kelley, J. K. Barton, Science 1999, 283, 375.

[16] O. F. A. Larsen, I. H. M. van Stokkum, B. Gobets, R. van Grondelle, H. van Amerongen, Biophys. J. 2001, 81, 1115.

[17] T. Fiebig, C. Wan, S. O. Kelley, J. K. Barton, A. H. Zewail, Proc. Natl. Acad. Sci. USA 1999, 96, 1187.

[18] C. Wan, T. Fiebig, S. O. Kelley, C. R. Treadway, J. K. Barton, A. H. Zewail, Proc. Natl. Acad. Sci. USA 1999, 96, 6014.

[19] The ps components for dTTP, dGTP, and dCTP were 10-40\% longer than in the corresponding complexes with Ap while for dATP a slightly shorter ps component ( $266 \mathrm{ps}$ ) was found. These differences can be attributed to variations in the complex structure. Only the Ap-R/dITP system did not exhibit the ps component.

[20] J. Smagowicz, K. L. Wierzchowki, J. Lumin. 1974, 8, 210.

[21] C. Reichhard, Solvent Effects in Organic Chemistry, VCH, Weinheim, 1988.

[22] A. Kettani, M. Gueron, J.-L. Leroy, J. Am. Chem. Soc. 1997, 119, 1108.

[23] E. C. Lim, J. Phys. Chem. 1986, 90, 6770.

[24] A. Holmen, B. Norden, B. Albinsson, J. Am. Chem. Soc. 1997, 119, 3114

[25] A. L. Sobelewski, W. Domcke, C. Dedonder-Lardeux, C. Jouvet, Phys. Chem. Chem. Phys. 2002, 4, 1093.

[26] T. M. Nordlund, D. G. Xu, K. O. Evans, Biochemistry 1993, 32, 1290.

[27] D.-G. Xu, T. M. Nordlund, Biophys. J. 2000, 78, 1042

[28] S. O. Kelley, J. K. Barton, Chem. Biol. 1998, 5, 413.

[29] S. Steenken, S. Jovanovic, J. Am. Chem. Soc. 1997, 119, 617.
[30] V. Shafirovich, A. Dourandin, N. P. Luneva, N. E. Geacintov, J. Chem. Soc., Perkin Trans. 2 2000, 2, 271.

[31] O. Schiemann, unpublished results.

[32] M. Enescu, L. Lindquist, J. Phys. Chem. 1995, 99, 8405.

[33] The small amplitude of the ps component and the fact that this component is absent when Ap is replaced by Ap-R shows that complexes with dITP are very sensitive to structural parameters and have weak binding energies. This observation is consistent with the small exothermicity that we propose for either hole or electron transfer from Ap* to I.

[34] C. A. M. Seidel, A. Schulz, M. H. M. Sauer, J. Phys. Chem. 1996, 100, 5541.

[35] N. S. Hush, A. S. Cheung, Chem. Phys. Lett. 1975, 34, 11.

[36] S. D. Wetmore, R. J. Boyd, L. A. Eriksson, Chem. Phys. Lett. 2000, 322, 129.

[37] K. Aflatooni, G. A. Gallup, P. D. Burrow, J. Phys. Chem. A 1998, 102, 6205.

[38] J. M. Jean, K. B. Hall, Proc. Natl. Acad. Sci. USA 2001, 98, 37.

[39] T. Yao, T. Wasa, S. Musha, Bull. Chem. Soc. Jpn. 1977, 50, 2917.

[40] J. Heinze, Angew. Chem. 1984, 96, 823; Angew. Chem. Int. Ed. 1984, $23,831$.

[41] V. Shafirovich, A. Dourandin, W. Huang, N. P. Luneva, N. E. Geacintov, J. Phys. Chem. B 1999, 103, 10924.

[42] R. A. Marcus, N. Sutin, Biochim. Biophys. Acta 1985, 811, 265.

[43] L. Eberson, Adv. Phys. Org. Chem. 1982, 18, 79.

[44] R. A. Kuharski, P. J. Rossky, J. Chem. Phys. 1985, 82, 5164.

[45] M. Cuma, S. Schneider, J. Phys. Org. Chem. 1997, 10, 383.

[46] D. O'Connor, V. Y. Shafirovich, N. E. Geacintov, J. Phys. Chem. 1994, 98, 9831.

[47] V. Y. Shafirovich, S. H. Courtney, N. Ya, N. E. Geacintov, J. Am. Chem. Soc. 1995, 117, 4920.

[48] Geometry optimizations were performed at the (U)B3LYP/6-31G* level of theory (without any symmetry restrictions) using Gaussian 94.

[49] H. Pal, Y. Nagasawa, K. Tominaga, K. Yoshihara, J. Phys. Chem. 1996, 100, 11964.

[50] J. Jortner, J. Chem. Phys. 1976, 64, 4860.

Received: April 12, 2002 [Z403] 